\title{
ANÁLISIS DE SUPERVIVENCIA DE IMPLANTES DENTALES EN LA DETERMINACIÓN DE SU ÉXITO 0 FRACASO, PRACTICADOS EN CONSULTORIOS DE LA CIUDAD DE PEREIRA, COLOMBIA
}

\author{
John Jairo Villa-Carmona', Diego Felipe Sandoval-Zuñiga², Santiago José \\ Jiménez-Moore ${ }^{3}$, Andrés Arismendi-Ramírez ${ }^{4}$
}

\section{Resumen}

Los implantes dentales han representado un gran avance en la odontología moderna y los esfuerzos realizados de la investigación científica y desarrollo industrial han permitido conseguir excelentes resultados de fiabilidad de las técnicas. El número de consultas odontológicas debido a pérdidas de piezas dentales ha arrojado como causas más comunes: edad, enfermedades bucofaríngeas que pueden conllevar a pérdida ósea, mala higiene bucal, deficiencias óseas, traumatismos, enfermedades sistémicas, entre otras. Como consecuencia de ello, los profesionales buscan permanentemente mejorar y diversificar los procesos tecnológicos de implantología; optimizan el tipo de material y los elementos usados en dichos procesos, con el afán de encontrar los mejores resultados de fijación y adaptación en sus pacientes. Esto les permite acceder a tecnología de punta con el fin de mejorar los resultados. El propósito de la investigación realizada es determinar el número de éxitos y fracasos en procesos de implantología en pacientes de consultorios odontológicos de la ciudad de Pereira, Colombia. Se realizó un estudio de tipo descriptivo, la caracterización de la técnica fue tipo de implantes. Se realizó una aplicación de ficha de recolección de datos a los odontólogos de los consultorios. Se determinó que el porcentaje de implantes exitosos corresponde al 97,1\%, de modo que la mayoría de los implantes son de tipo largo con un porcentaje del 94,58\%. No se identificó que exista relación directa entre el fracaso del implante y el tipo de consultorio.

Palabras clave: oseointegración, implantes dentales, carga inmediata en implantología oral.

\footnotetext{
1John Jairo Villa-Carmona. jvilla20@areandina.edu.co". Licenciado en Biología y Química. Especialista en Biología Molecular y Biotecnología. (c) M.Sc. Biología Molecular y Biotecnología. Profesor Facultad de Ingeniería y Ciencias Básicas, Fundación Universitaria del Área Andina, Pereira.

${ }^{2}$ Biólogo. Especialista en Pedagogía y Docencia. (c) M.Sc Biología Molecular y Biotecnología. Profesor Fundación Universitaria del Área Andina, Pereira.

${ }^{3}$ Odontólogo, Rehabilitador Oral. Profesor Fundación Universitaria del Área Andina, Pereira. ${ }^{4}$ Ingeniero físico. Especialista en Pedagogía y Docencia. (c) M.Sc. Ciencia de los Datos y Procesamiento de Datos Masivos (Big Data). Profesor Fundación Universitaria del Área Andina, Pereira.
} 


\section{SURVIVAL ANALYSIS OF DENTAL IMPLANTS TO DETERMINE THEIR SUCCESS OR FAILURE, DEVELOPED IN DENTAL OFFICES OF THE CITY OF PEREIRA, COLOMBIA}

John Jairo Villa-Carmona', Diego Felipe Sandoval-Zuñiga², Santiago José

Jiménez-Moore ${ }^{3}$, Andrés Arismendi-Ramírez ${ }^{4}$

\section{Abstract}

Dental implants have represented a great advance in modern dentistry. The efforts made in scientific research and industrial development have allowed to achieve excellent results regarding reliability of the techniques. The amount of patients consulting the dentist due to the loss of teeth, have shown that the main causes are, age, oropharyngeal diseases that can produce bone loss, poor oral hygiene, bone deficiencies, trauma, systemic diseases, among others. As a result, dental professionals permanently seek to improve and diversify the technological implant processes, optimizing the type of material and tools used in these processes, with the purpose of achieving the best results of fixation and adaptation for patients; which allows them to access to the latest technology, and have the best results. The purpose of this research is to determine the amount of success and failure of dental implants in patients of dental offices in the city of Pereira, Colombia. A descriptive study was carried out, the characterization of the technique, type of implants; support the research by applying to the dentists an instrument of data collection. It was determined that the percentage of successful implants corresponds to $97.1 \%$. Evidencing that most of the implants are long type $94.58 \%$ respectively, it was not stablished that there is a direct relationship between the failure of the implant and the type of dentist office. 


\section{ANÁLISE DE SOBREVIVÊNCIA DE IMPLANTES DENTÁRIOS PARA DETERMINAR O SEU SUCESSO OU FRACASSO, EFETUADOS EM CONSULTÓRIOS DA CIDADE DE PEREIRA, COLÔMBIA}

John Jairo Villa-Carmona', Diego Felipe Sandoval-Zuñiga², Santiago José Jiménez-Moore ${ }^{3}$, Andrés Arismendi-Ramírez ${ }^{4}$

\section{Resumo}

Os implantes dentários têm representado um avanço na odontologia moderna. Os esforços de investigação científica e desenvolvimento industrial tornaram possível alcançar excelentes resultados de confiabilidade das técnicas. $\mathrm{O}$ número de visitas ao dentista devido à perda de dentes, têm como causas mais comuns: idade, doenças da orofaringe que podem levar à perda óssea, má higiene oral, deficiência óssea, trauma, doenças sistêmicas, entre outros. Como resultado, os profissionais estão constantemente procurando melhorar e diversificar os processos tecnológicos de implantologia, otimizando o tipo de material utilizado nestes processos, com o objetivo de encontrar os melhores resultados de fixação e adaptação para os pacientes; o que lhes permite acessar tecnologia de ponta, a fim de melhorar os resultados. O objetivo da pesquisa feita é determinar o número de sucessos e falhas nos processos de implantologia em pacientes de clínicas odontológicas na cidade de Pereira, Colômbia. Foi realizado um estudo descritivo, a caracterização da técnica, tipo de implantes, suportando a pesquisa mediante a aplicação de um formulário de coleta de dados para os dentistas dos consultórios. Foi determinado que a porcentagem de implantes de sucesso corresponde a 97,1\%. É evidenciando que a maioria dos implantes são de tipo longo, com uma percentagem de $94,58 \%$. Não é identificada relação direta entre a falha do implante e o tipo de consultório. 


\section{Introducción}

Los dientes son trascendentales para desarrollar una vida normal. Su función principal es triturar los alimentos para favorecer una correcta digestión, además de su importancia en la fonación y la expresión armoniosa de la cara. Por ello, juegan un rol importante en el desarrollo y desempeño social de una persona; una buena dentadura demuestra signos de salud y bienestar (1). La salud bucal no está relacionada solamente con la presencia de los dientes, también con el estado de los tejidos blandos en la cavidad oral, los cuales no deben perturbar la capacidad y la salud general del individuo (2).

Uno de los problemas que afecta la salud oral es el edentulismo (ausencia dental parcial o total), que no es necesariamente imputable a la vejez, dado que existen múltiples causas de la pérdida dentaria como, por ejemplo: caries, periodontopatias, maloclusiones(3), pérdida de masa ósea, disminución ósea e incremento de la porosidad, entre otras causas, tales como las enfermedades sistémicas, accidentes, molestias óseas, traumas dentoalveolares y la extracción de piezas dentales. La permanencia o pérdida de dichas estructuras puede afectar emocional y socialmente a las personas (4).

Al momento de su pérdida, los pacientes buscan a los profesionales de la salud oral, y obtienen la opción de implantes dentales como principal procedimiento terapéutico para la rehabilitación oral, dado que los implantes permiten la reposición de dientes perdidos. El proceso que hace posible su fijación se conoce como oseointegración, que se puede definir como fase de unión entre los implantes dentales y el hueso recientemente formado (5). Dicho procedimiento es la respuesta a la gran demanda de personas con edentulismo parcial o total con altas tasas de éxito y notoria satisfacción para los pacientes (6).

Para los distintos procedimientos de implantología, la utilización de implantes de longitudes cortas y largas pueden tener diferentes tratamientos, y generan gran expectativa en el éxito o fracaso del implante (7). Los criterios comúnmente aceptados para la evaluación del éxito del implante fueron propuestos por Albrektsson et al (8) para identificar evidencia clínica de osteointegración exitosa y supervivencia de los implantes. En las últimas tres décadas, el éxito del implante ha sido evaluado por las tasas de supervivencia, la estabilidad continua de la prótesis, la pérdida ósea radiográfica y la ausencia de infección en los tejidos blandos periimplantarios $(8,9,10,11,12,13)$.

Las técnicas son también indispensables para la buena fijación de los implantes dentales, por ejemplo, los tratamientos superficiales se emplean para obtener aumento de la rugosidad superficial. De este modo, se favorece la oseointegración de los implantes a corto y mediano plazo, pues beneficia la adhesión celular. Este efecto tiene como consecuencia una rápida regeneración y una mejor calidad del tejido óseo (14). Actualmente, algunos autores estiman que el fracaso de los implantes después del proceso de oseointegración se debe fundamen- 
talmente a ciertas condiciones biológicas, como la infección bacteriana, y no al rechazo cuando se colocan implantes de calidad contrastada, aunque también se atribuye a características específicas de los pacientes y a la destreza de los cirujanos(15). También se asumen fallas mecánicas, tales como la fractura de un implante, el deterioro de su conexión protésica y la presencia de un tornillo protésico fracturado dentro del implante sin que sea posible retirarlo de su interior, lo que inutiliza el implante. También se asumen fallas humanas, que se refieren a posicionamientos de implantes en sitios intraorales con imposibilidad de rehabilitación (16).

El propósito del estudio fue la caracterización descriptiva y estadística de las técnicas y tipos de implantes en los procesos de implantología oral en pacientes de consultorios odontológicos de la ciudad de Pereira, Colombia. Las variables evaluadas se basaron en: éxito o fracaso, tipo de implante, tipo de carga, causas de fracaso del implante en estos procedimientos.

\section{Materiales y métodos}

Se realizó una investigación de tipo descriptiva, de corte transversal en 5 clínicas odontológicas privadas de la ciudad de Pereira, durante el transcurso del año 2015. El criterio de inclusión se determinó según los procesos de inserción de implantes dentales realizados en dichos consultorios, así como la aceptación después de la invitación formal (cartas) que se realizó por parte de los investigadores a los consultorios odontológicos de la ciudad.
La población tomada en el estudio fueron los implantes dentales realizados en la ciudad de Pereira; la muestra fue tomada de los consultorios en mención donde se lleva a cabo este tipo de procedimientos, teniendo en cuenta que no todos los consultorios tenían permitido realizarlos. No se establecieron las condiciones sociodemográficas de los pacientes, ya que no se tuvo acceso a los datos personales, puesto que la historia clínica es un documento privado de tipo técnico, clínico, legal, obligatorio y sometido a reserva.

El estudio abarcó la totalidad de pacientes, sin tener contacto directo con los mismos, ni sus historias clínicas, las cuales fueron el soporte del profesional para suministrar la información que reunía los criterios en mención, es decir, con prótesis rehabilitadas para su inclusión. Se obtuvo un total de 683 implantes oseointegrados y su información fue suministrada por cada especialista desde sus bases de datos. Por esta razón, no se necesitó aval de un comité de ética.

\section{Diseño de la ficha de recolección de datos}

La ficha de recolección de datos se diseñó para obtener información de la caracterización de los procesos de implantes oseointegrados que se practican en los pacientes en los consultorios odontológicos de la ciudad de Pereira, reseñados anteriormente. Las preguntas se enfocaron en éxitos o fracasos de estos procesos, tipos de implantes y las técnicas utilizadas en los procedimientos. 


\section{Aplicación de la matriz de recolección Resultados de datos}

La aplicación de la ficha de recolección de datos se realizó mediante la visita a cada uno de los consultorios odontológicos. Los profesionales suministraron información basándose en las historias clínicas $y$, de esta manera, dieron respuestas a las preguntas del cuestionario, el cual era diligenciado por uno de los investigadores. Los datos que se presentan fueron suministrados desde el año 2012 al 2015.

\section{Procesamiento de los datos}

Los datos obtenidos se procesaron en una matriz realizada en Microsoft Excel 2010 y se enlazaron con el programa IBM SPSS STATISTICS V.24 para el tratamiento estadístico descriptivo.

El total de implantes realizados por los consultorios odontológicos que participaron en el estudio es de 683 de los cuales fueron exitosos 663, que corresponden al 97,1\%; los 20 fallidos corresponden al 2,9\%. La Tabla 1 muestra los implantes exitosos y fallidos clasificados por los diferentes consultorios.

En el gráfico 1 se puede notar el número de implantes por consultorios, así como la cantidad de implantes exitosos y fallidos de cada uno de los consultorios odontológicos pertenecientes al estudio. Allí, se evidencia el bajo porcentaje de implantes fallidos; sin embargo, cabe resaltar que ninguno de los consultorios tiene una efectividad del $100 \%$ en este tipo de procesos.

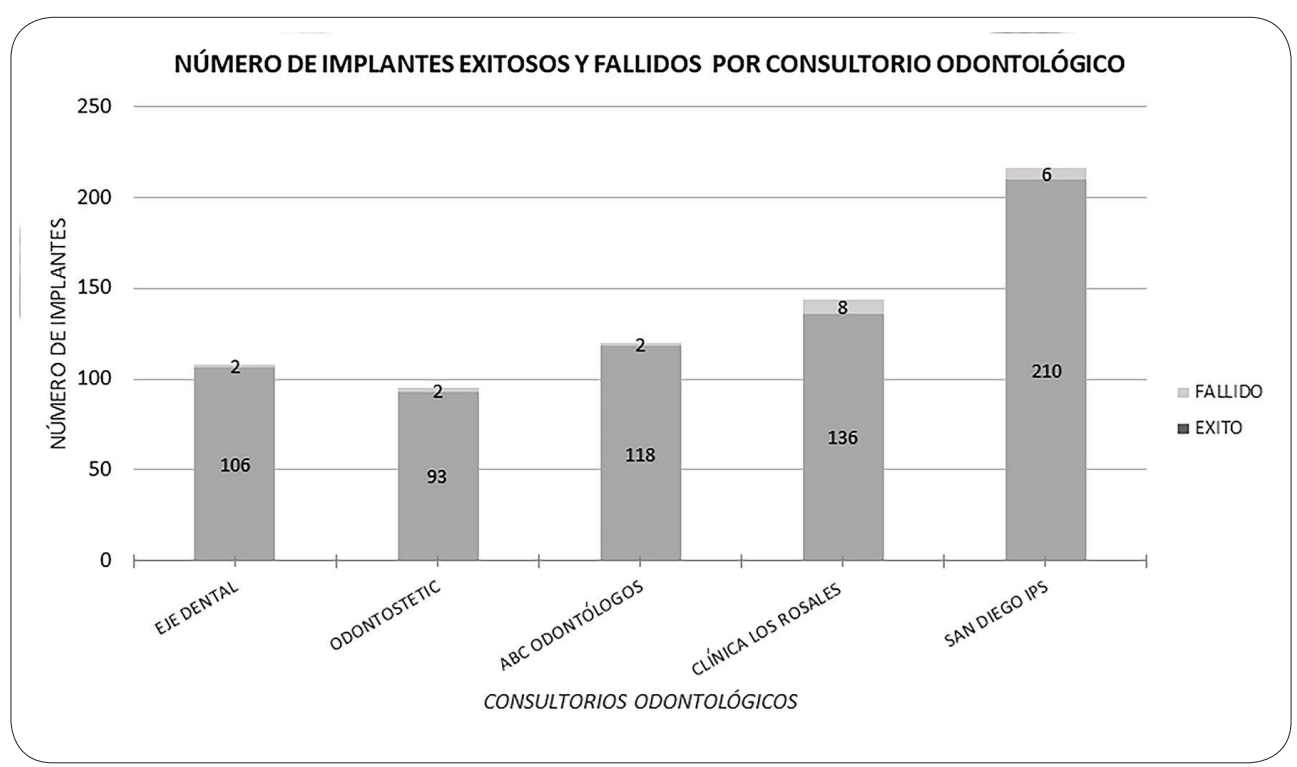

Figura 1. Número de implantes exitosos y fallidos por consultorio odontológico 
Tabla 1. Número de implantes exitosos y fallidos por consultorio odontológico

\begin{tabular}{l|c|r|r}
\hline \multicolumn{4}{c}{ Pruebas de chi-cuadrado } \\
\hline & Valor & df & $\begin{array}{r}\text { Significación } \\
\text { asintótica } \\
\text { (bilateral) }\end{array}$ \\
\hline Chi-cuadrado de Pearson & $4,853 a$ & 4 &, 303 \\
Razón de verosimilutid & 4,349 & 4 &, 361 \\
N de casos válidos & 683 & & \\
\hline
\end{tabular}

Fuente: elaboración propia.

Para el análisis estadístico, se formuló la prueba de Chi cuadrado de Pearson (X2) (Tabla 1) cuyo fin es hacer un contraste estadístico de la relación de dependencia de las variables cualitativas del consultorio y éxito o fracaso en los implantes. Se determinó trabajar con un intervalo de confianza de $95 \%$, es decir, el $\alpha=0,05$, el valor de chi-cuadrado es 4,853; el grado de significancia es $\mathrm{p}=0,303$; como $\mathrm{p}>0,05$, se considera que no hay relación entre el consultorio y el éxito o fracaso del implante.

En contraste, se relacionó el éxito y el fracaso con la longitud de los implantes usados. Por ende, se considera una variable importante en los procesos de implantología oral. Para el estudio se consideró como implante largo aquellos que presentan longitud mayor o igual a $10 \mathrm{~mm}$, mientras que los implantes cortos presentan longitud menor de $10 \mathrm{~mm}$ (17).

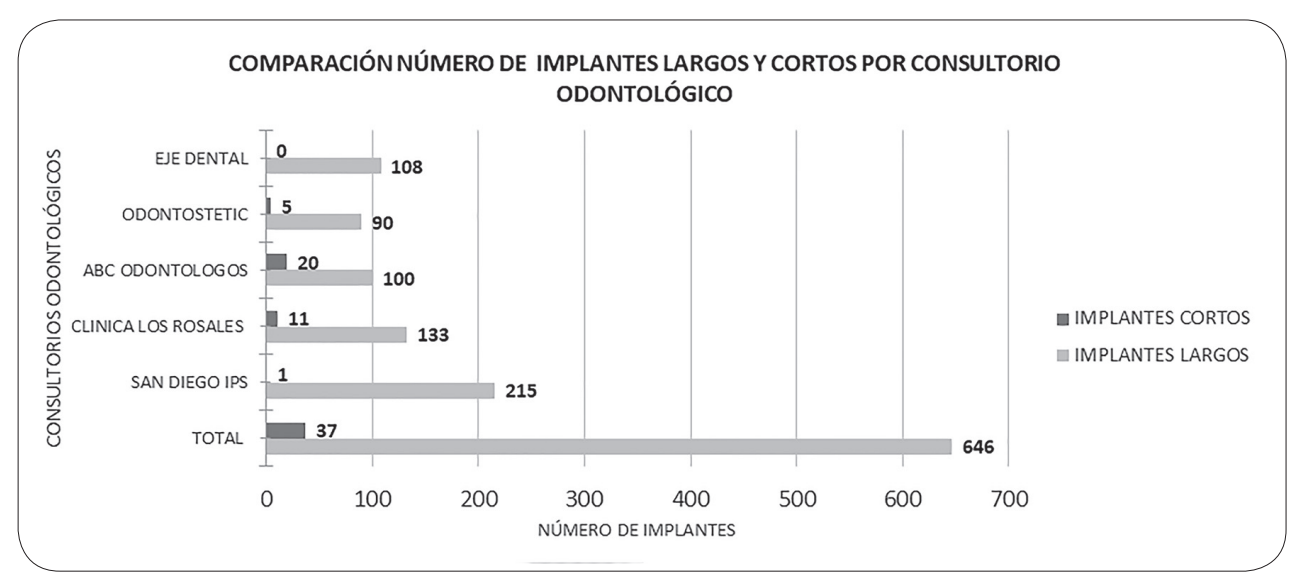

Figura 2. Comparación número de implantes largos y cortos por consultorio odontológico Fuente: elaboración propia. 
En el Gráfico 2, se evidencia que los diferentes consultorios optan principalmente por el uso de implantes largos, así que es evidente la prevalencia por los implantes de este tipo, dado que 646 casos, correspondientes al 94,6\%, tenían como longitud mayor o igual a $10 \mathrm{~mm}$ o más; mientras que solo 37 implantes, equivalentes al $5,4 \%$, presentaban longitud menor de $10 \mathrm{~mm}$.

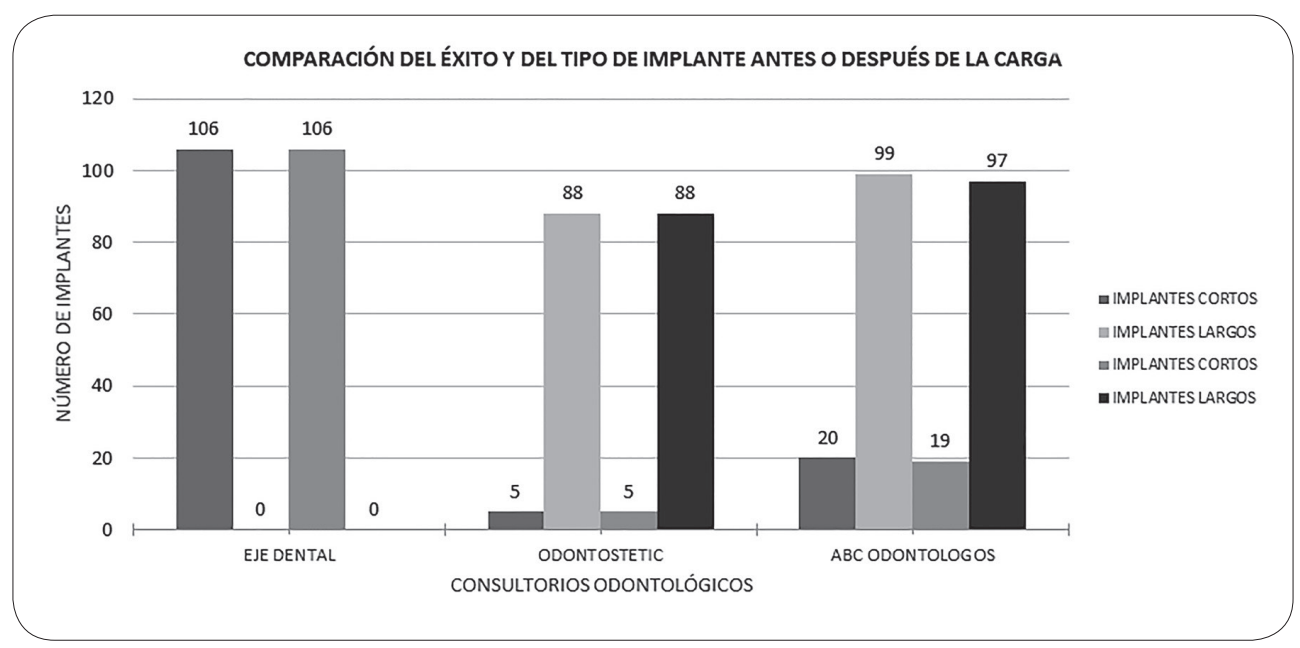

Figura 3. Comparación del éxito del tipo de implante antes o después de la carga Fuente: elaboración propia.

En el estudio se determinó el éxito del procedimiento de implantología oral, que se puede clasificar en antes o después de la carga, al igual que con la longitud del implante. El gráfico 3 muestra los resultados concernientes solamente a tres laboratorios, ya que estos fueron los que aportaron esta información, los demás consultorios no tenían especificado este ítem en sus historias clínicas.

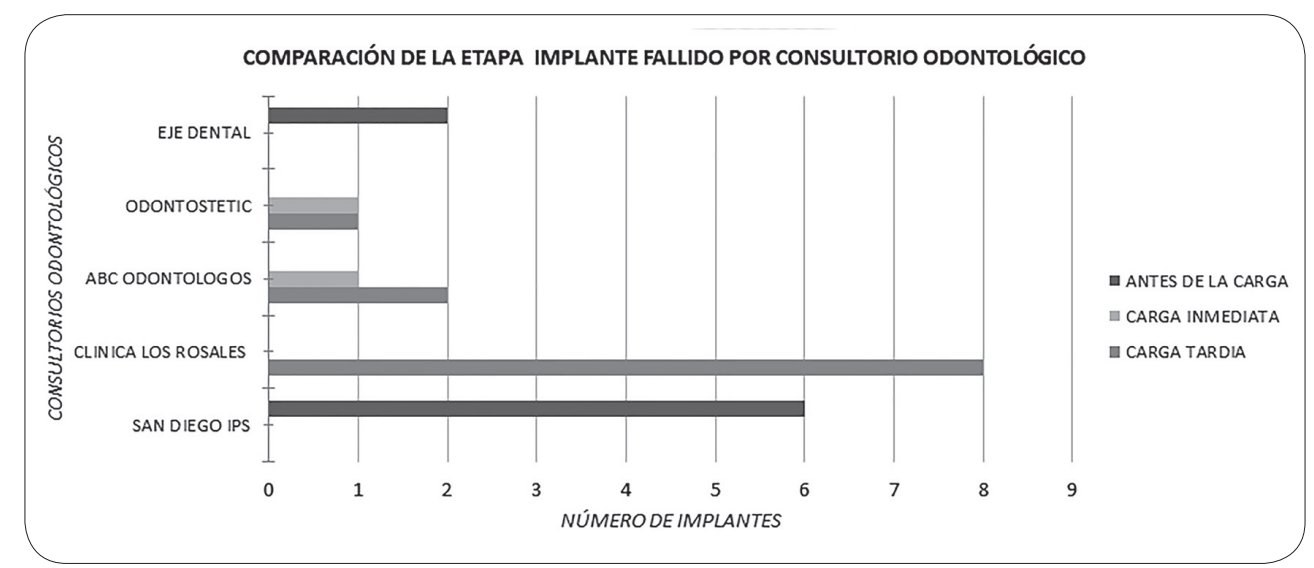

Figura 4. Comparación del tipo de carga del implante fallido por consultorio odontológico. Fuente: elaboración propia. 
El éxito de la implantología depende de muchos factores y uno de ellos es el control de las cargas; no depende exclusivamente en realizar un protocolo quirúrgico y protésico correcto y mantener los tejidos periimplantarios sanos, sino de los principios biomecánicos y de oclusión. Estos últimos cobran importancia en la carga inmediata por la ausencia de contacto en posición de máxima intercuspidación y en movimientos anormales (18). La colocación de implantes de carga inmediata se considera una opción ventajosa para la rehabilitación con prótesis dentales (19). En el gráfico 4 se establece el tipo de carga en la cual falló los implantes para cada uno de los consultorios odontológicos.

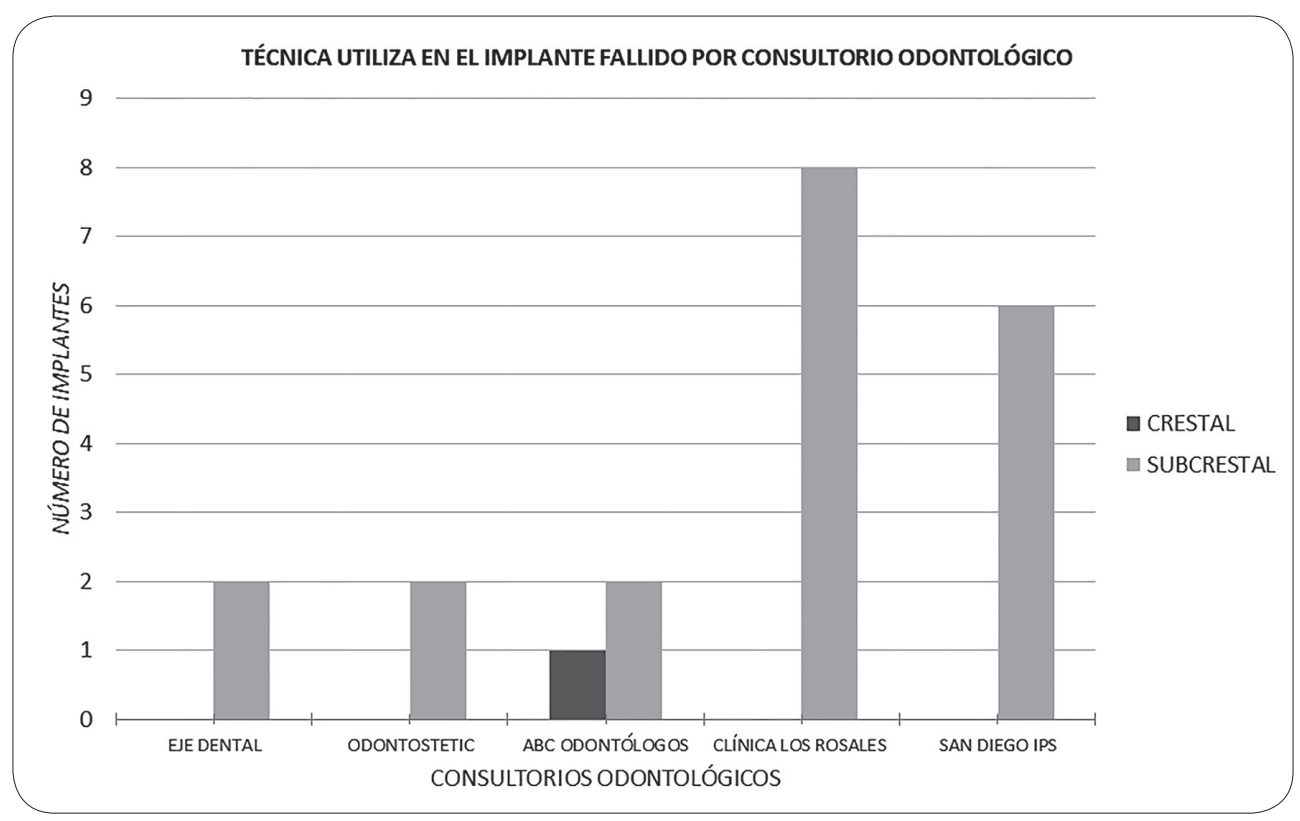

Figura 5. Técnica utilizada en el implante fallido por consultorio odontológico. Fuente: elaboración propia.

La implantología oral ha revolucionado la práctica odontológica y ha tenido un rápido desarrollo. Después de la evidencia científica de la posibilidad con éxito de la utilización de los implantes y del conocimiento de los fenómenos biológicos de la oseointegración, la tendencia ha sido hacia la integración simplificada de la técnica quirúrgica (20). Estos procedimientos se pueden dividir en técnica crestal y técnica subcrestal. Se determinó en el estudio la técnica realizada en el implante fallido (datos represen- tados en el Gráfico 5). Es evidente que en los implantes fallidos la técnica más utilizada en cada uno de los consultorios es la subcrestal.

Las fallas biológicas podrían ser consideradas todas las veces en que hubiera insuficiencia del huésped en establecer o mantener la oseointegración, que puede ser considerada como una falla precoz, por no poder mantenerse este fenómeno cuando el implante es sometido a 
ocurren cuando la oseointegración no se mantiene después de la instalación de los implantes o cuando no es mantenida a través de los años (21).

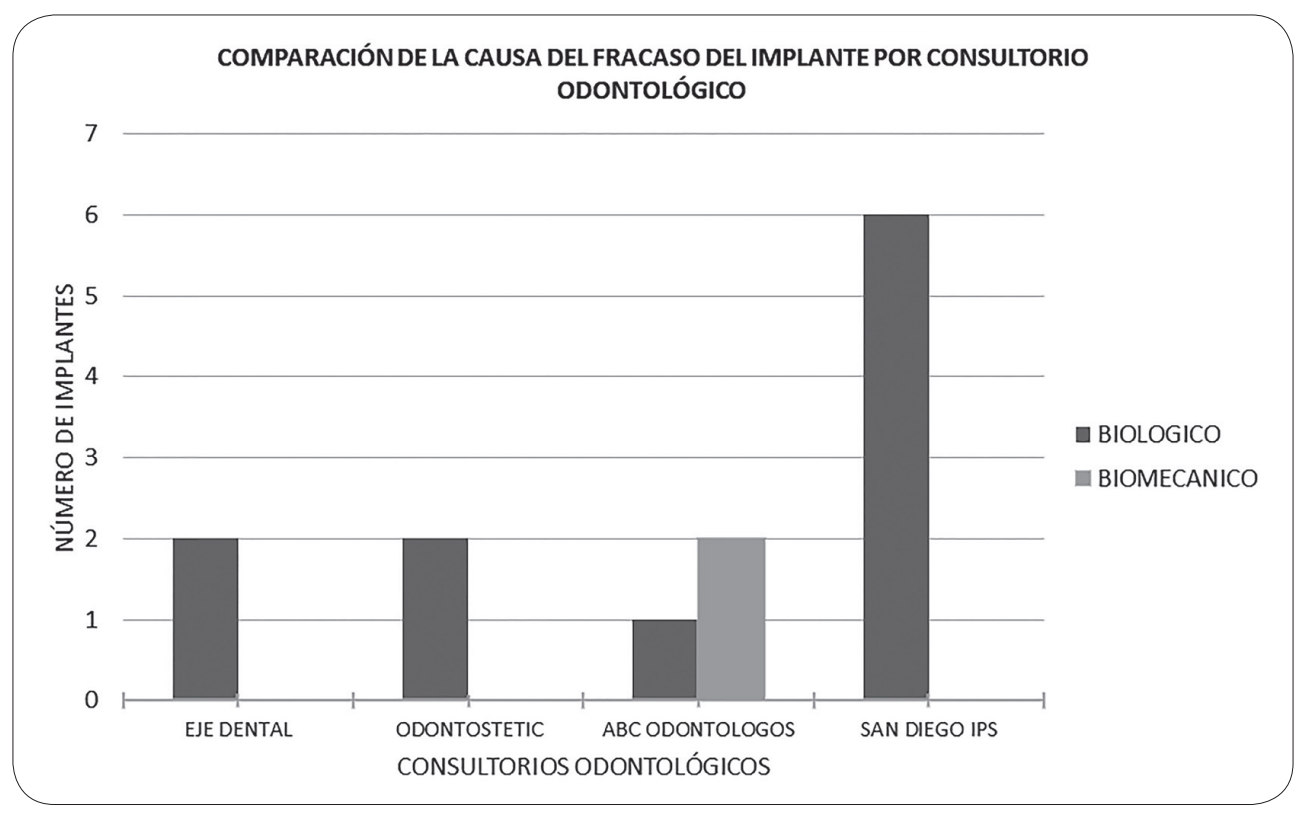

Figura 6. Comparación de las causas del fracaso del implante por consultorio odontológico. Fuente: elaboración propia.

De los pacientes con implantes considerados fallidos, se establecieron las causas del fracaso. Por esta razón, se clasificaron como motivos biológicos y motivos biomecánicos. Los resultados por consultorio se muestran en el Gráfico 6 , donde se nota que, principalmente, los implantes fallidos son por motivos biológicos, dado que 11 fueron por este motivo, mientras que solo fueron 2 por motivos biomecánicos.

\section{Discusión}

El presente estudio se caracterizó por diferentes variables asociadas a los procesos de implantes de piezas dentales

110 que tienen relevancia en el éxito o fracaso de estos procedimientos. Como se menciona al principio de este documento, el éxito quirúrgico de los implantes está directamente relacionado con un proceso de interacción con el hueso (osteointegración); no obstante, cuando el procedimiento ha fallado, se presenta una alteración durante la fase inicial y se produce una formación de tejido cicatricial fibroso entre la superficie del implante y el hueso circundante (22). Se sabe que son muchos los factores que pueden contribuir al éxito o al fracaso de los implantes, desde la condición del paciente hasta los protocolos quirúrgicos y protésicos realizados por el operador (23). La falla del implante es más fácil de describir que el éxito o supervivencia del implante y puede consistir en una variedad de factores. Cualquier dolor, movi- 
lidad vertical y pérdida ósea progresiva no controlada justifican la extracción del implante (16).

Se determinó que el porcentaje de implantes exitosos corresponde al $97,1 \%$; el de implantes fallidos, 2,9\%. Esto no difiere de los porcentajes de los artículos que fueron tomados como antecedentes, los cuales están en un rango entre el 90\% al $98 \%$ en el caso de los éxitos y el $1 \%$ al $5 \%$ de los casos de los fracasos. Se exponen algunos factores que afectan el éxito del proceso de implantología, tales como: el hábito de fumar, la deficiente higiene bucal, la sobrecarga protésica, los hábitos parafuncionales (bruxismo), la edad avanzada del paciente; además, la disminución de la irrigación sanguínea en el sitio receptor del implante; la ubicación del implante dental relacionada con la calidad y cantidad ósea del sitio receptor; al igual que las técnicas quirúrgicas para lograr un aumento de la cantidad ósea, entre otros (24).

En el caso de la variable tamaño del implante se evidenció que la mayoría de los implantes son de tipo largo, con un porcentaje del 94,58\%. Los implantes cortos obtuvieron un porcentaje de 5,42\%. El tamaño de los implantes (longitud y diámetro) puede también influir en el éxito del implante. Por cada $3 \mathrm{~mm}$ de longitud, el área de superficie se incrementa un 20-30\%. Además, los fracasos son superiores cuando se utilizan implantes con una longitud menor de $10 \mathrm{~mm}$ (25). En este sentido, el tamaño del implante es todavía más relevante en los casos de mala calidad ósea. Por otro lado, la variable tipo de carga refiere a un protocolo clínico para la colocación y aplicación de fuerzas sobre los implantes mediante la rehabilitación fija o removible. La carga inmediata es aquella que se realiza en la misma sesión clínica o dentro de las 48 horas siguientes, y en el estudio se estableció que el mayor porcentaje de implantes orales fallidos se presentó con la técnica de carga tardía. De igual manera, la técnica quirúrgica crestal es la que prevalece en los implantes fallidos (26).

\section{Conflicto de intereses}

Los autores declaran no tener ningún conflicto de intereses. En el momento de la recolección de datos, el profesional en odontología de cada consultorio que participó en la investigación suministró la información a partir de la base de datos; aclaramos que en este proceso los investigadores no tuvieron contacto con los pacientes, adquiriendo información general, sin acceder a la base de datos del consultorio específica (historias clínicas).

\section{Agradecimientos}

Este trabajo pudo ser escrito gracias al apoyo y colaboración de:

Fundación Universitaria del Área Andina.

Las clínicas odontológicas: Eje dental, centro de ortodoncia y cosmetología oral; San Diego IPS odontología; Clínica Odontológica Odontostetic 
de Colombia S.A.; ABC odontólogos especialistas; Consultorio Odontólogico en el edificio Clínica los Rosales en la ciudad de Pereira, Colombia.

\section{Referencias}

1. Gonzalez R. Origen y evolución de los implantes dentales. Rev haban cienc méd. 2009;8(4):9.

2. González R, Madrazo D, Osorio M. Conocimientos sobre salud bucal en los círculos de abuelos. Rev Cubana Estomatol. 2013;50(3):284-91.

3. Trujillo D, Isabel I, Brito M, René I. Injertos óseos en implantología oral. Rev Medica Electron. 2014;36(4):449-61.

4. Lorenzo S, Piccardo V, Alvarez F, et al. Enfermedad Periodontal en la población joven y adulta uruguaya del Interior del país. Relevamiento Nacional 20102011. Odontoestomatologia. 2013;XV. (Noespecial):35-46.

5. García E, et al. La rehabilitación protésica unitaria sobre implantes en el paciente parcialmente desdentado. Rev Ciencias Médicas. 2015;19(3):403-12.

6. González Y, Castillo E, Mesa B. Comportamiento del proceso de osteointegración en implantes transalveolares inmediatos. MediSur. 2003;14(1):26-33.

7. Rodríguez M. Supervivencia de implantes cortos, menores o iguales a 8 $\mathrm{mm}$, de superficie arenada y grabada con ácido. Claves odontol. 2014;21(73):49-66.

8. Alberktsson T. The Long-Term Efficacy of Currently Used Dental Implants. Int J Oral Maxillofac Implant. 1997;11-25.
Juliana Ospina López, estudiante odontología quinto semestre. Fundación Universitaria del Área Andina, seccional Pereira.

9. Smith D, Zarb G. Criteria for success of osseointegrated endosseous implants. J Prosthet Dent. 1989;62(5):567-72.

10. Buser D, Weber H, Lang N. Tissue integration of non-submerged implants. Inst Straumann. 1990;1(1):33-40

11. Misch C, et al. Implant success, survival, and failure: The International Congress of Oral Implantologists (ICOI) pisa consensus conference. Implant Dent. 2008;17(1):5-15.

12. Annibali S, Bignozzi I, La Monaca $G$, Cristalli M. Usefulness of the Aesthetic Result as a Success Criterion for Implant Therapy: A Review. Clin Implant Dent Relat Res [Internet]. 2012 [citado 2018 ago 27];14(1):340. Disponible en: http://doi.wiley. com/10.1111/j.1708-8208.2009.00234.x

13. Albrektsson T, Zarb G. Determinants of correct clinical reporting. Int J Prosthodont [Internet]. 1998 [citado 2018 sep 10];11(5):517-21. Disponible en: http:// www.ncbi.nlm.nih.gov/pubmed/9922743

14. Rodríguez D, García F. Physicochemical characterization of the surface of 9 dental implants with 3 different surface treatments. Med Oral Patol Oral Cir Bucal. 2005;10(1):58-65.

15. Corona $\mathrm{M}$, et al. Main factors provoquing dental implant failure Dra. Marcia Hortensia Corona Carpio. Medisan. 2015;19(11):1325-9. 
16. Leighton $Y$, Carvajal C. Evaluación de un nuevo método de remoción quirúrgica de implantes oseointegrados considerados en fallo. Rev Clínica Periodoncia, Implantol y Rehabil Oral [Internet]. 2015 [citado 2018 ago 27];8(1):45-53. Disponible en: http://linkinghub.elsevier.com/retrieve/pii/ S0718539115000129

17. Pérez $\mathrm{O}$, Velasco $E$, Rodríguez $\mathrm{O}$, González L. Resultados del tratamiento con implantes dentales osteointegrados en la fase quirúrgica. Rev Cubana Estomatol. 2013;50(4):351-63.

18. Eleuteria $M$, Torres $M$, Vigoa D. Rehabilitación protésica combinando implante-diente natural. Rev Ciencias Médicas. 2013;17(5).

19. Santos S, Cruz M, Córdova D. Tratamiento con implantes LeaderNano en paciente con oligodoncia. Rev Electrónica Dr Zoilo E Mar Vidaurreta. 2015;40.

20. Velasco E, et al. La expansión ósea en la implantología oral. Av en Periodoncia e Implantol Oral. 2008;20(2):95-101.
21. Cruz L. Fallas mecánicas y biológicas en las prótesis sobre implantes. Ciencias Médicas. 2012;11(4):572.

22. Esposito M, Thomsen P, Ericson L, Lekholm U. Histopathologic Observations on Early Oral Implant Failures. Int J Oral Maxillofac Implants. 2000;14(6):798-810.

23. Implants $\mathrm{M}$. Oral \& maxillofacial implants. Int J Oral y Maxillofac Implant. 2011;26.

24. Seminario $M$, Morgenstern $H$. Prevalencia de la pérdida de implantes dentales en una Facultad Peruana de Odontología, entre los años 2001 y 2011. 2012;9(2):119-24.

25. Peniche R. Consideraciones quirúrgico-protésicas para la carga oclusal inmediata en implantología oral. Adm. 2011;68(4):161-8.

26. Herrera $M$, et al. Supervivencia de implantes dentales entre la primera y la segunda fase quirúrgica. Rev Arch Médico Camagüey. 2013;17(3):278-88. 
INVESTIGACIONES ANDINA No. 37, Vol. 20 